\title{
Variables que predicen la participación en los dispositivos de apoyos académicos institucionales (AAI)
}

\section{Jorge Valenzuela ${ }^{1}$, Jorge Miranda-Ossandón ${ }^{2}$, Álvaro González ${ }^{3}$, Marcelo González ${ }^{4}$, Pablo Cáceres ${ }^{5,}$ Carla Muñoz ${ }^{6}$}

${ }^{1}$ Facultad de Ciencias de la Educación, Universidad Católica del Maule, Talca

${ }^{2}$ Departamento de Ciencias de la Educación, Facultad de Educación, Universidad Católica de Temuco, Temuco

${ }^{3}$ Departamento de Educación, Universidad de Magallanes, Punta Arenas

${ }^{4}$ Equipo Interuniversitario de Investigadores en educación, Santiago

${ }^{5}$ Escuela de Psicología, Pontificia Universidad Católica de Valparaíso, Viña del Mar

${ }^{6}$ Departamento de Psicología, Universidad Católica del Maule, Talca

\section{Chile}

Correspondencia: Jorge Valenzuela, Facultad de Ciencias de la Educación, Universidad Católica del Maule, Av. San Miguel 3606, Talca, Chile E-mail: jvalenzuela@ucm.cl

(C) Universidad de Almería and Ilustre Colegio Oficial de la Psicología de Andalucía Oriental (Spain) 


\section{Resumen}

Introducción: Ante la necesidad de focalizar los esfuerzos para que los estudiantes que más lo requieran puedan ser parte de los apoyos académicos que brindan las instituciones universitarias, se realizó un estudio para identificar las variables que mejor predicen la participación voluntaria en estos dispositivos.

Método: Participaron en este estudio 803 estudiantes universitarios de ambos sexos de cuatro universidades chilenas. El instrumento midió variables de carácter personal y condicionantes relacionadas con la búsqueda de ayuda académica en la Educación Superior. Los datos fueron analizados por medio de una regresión logística binomial.

Resultados: Los resultados permiten identificar como variables predictoras de la participación en dispositivos de apoyos académicos institucionales (AAI), a la conciencia de necesidad de ayuda, al conocimiento de los procedimientos concretos para acceder a estos AAI, a la experiencia de búsqueda de ayuda en general, así como a la variable costo asociada a la pérdida de alternativas valiosas.

Discusión y conclusiones: Tras la identificación de las variables que predicen la participación en los dispositivos de apoyos académicos ofertados por las instituciones universitarias, se discuten los alcances de estos resultados.

Palabras Clave: búsqueda de ayuda, apoyos académicos institucionales, variables motivacionales, variables inhibitorias 


\begin{abstract}
Introduction: Given the need to focus efforts so that students who most require it can be part of the academic support provided by university institutions, a study was conducted to identify the variables that best predicts voluntary participation in these services.

Method: A total of 803 male and female university students from four Chilean universities participated in this study. The instrument measured personal and conditioning variables related to academic help-seeking in higher education. The data were analyzed through of binomial logistic regression.
\end{abstract}

Results: The results allow us to identify as predictor variables of participation in institutional academic support services (IAS) the awareness of the need for help, the knowledge of the specific procedures to access these IAS, the experience of seeking help in general, as well as the cost variable associated with the loss of valuable alternatives.

Discussion or Conclusion: After identifying the variables that predict participation in the academic support offered by universities, the scope of these results is discussed

Keywords: help-seeking, institutional academic support, motivational variables, Inhibitory variables 


\section{Introducción}

Existe una amplia literatura acerca del proceso de búsqueda de ayuda académica, en los distintos niveles educativos y en diferentes modalidades. Sin embargo, son escasos los estudios que den cuenta de este proceso cuando la fuente de ayuda que se busca corresponde a un dispositivo de tipo institucional, particularmente en el caso de la educación terciaria (Collins y Sims, 2006).

De manera coincidente con la literatura reciente, reportes informales de distintos actores que brindan apoyos académicos a estudiantes universitarios muestran que uno de los problemas asociados a los apoyos académicos institucionales (AAI) es que los estudiantes que aprovechan estas instancias, por diversas razones, no son precisamente aquellos que más lo necesitan (Finney et al., 2018; Peeters et al., 2020; Ryan et al., 2005; Seamark y Gabriel, 2018), y que cuando este grupo de estudiantes accede a ellos, es generalmente muy tarde, imposibilitando que los esfuerzos institucionales logren la efectividad esperada.

En Chile, prácticamente la totalidad de las universidades chilenas poseen sistemas de apoyo de diverso orden (Frites y Molina, 2016; Miranda et al., 2016; Ordenes et al., 2018; Venegas y Gairín, 2020). Sin embargo, al igual que en otras latitudes, no siempre los apoyos son aprovechados por quienes más lo necesitan (Collins y Sims, 2006) y su frecuencia de uso depende de la conveniencia, más que de la utilidad de dicho apoyo (Wirtz et al., 2018). No obstante, desconocemos si las variables que la literatura reporta y que están asociadas a la búsqueda de ayuda en general, son a la vez aplicables en un contexto donde la ayuda proviene desde la institución universitaria.

En este contexto, comprender las variables que explican que los estudiantes se dispongan psicológicamente a aprovechar los apoyos académicos que brinda la institución y que finalmente concreten su participación en estos dispositivos, constituye un conocimiento necesario, tanto para potenciar las variables que favorecen esta conducta, como para reducir el impacto de aquellas que actúan de manera inhibitoria. 


\section{Búsqueda de apoyo académico}

Los primeros trabajos en torno a la búsqueda de ayuda surgen en la década de los ochenta de la mano de la investigación desarrollada por Nelson-Le Gall (1981), quien plantea que la búsqueda de ayuda es una estrategia de aprendizaje susceptible de ser modelizada como un proceso sistemático, constituido de diferentes etapas o fases.

Desde los trabajos pioneros de Nelson-Le Gall (1981), se han identificado factores que favorecen o inhiben la búsqueda de ayuda y variables que inciden en cada una de las etapas del proceso, enriqueciendo con ello el modelo teórico propuesto (Karabenick y Berger, 2013; Puustinen, 2013; Reeves, 2014). Hoy, la búsqueda de ayuda es ampliamente comprendida como una estrategia de aprendizaje autorregulado (Karabenick y Gonida, 2018; Newman, 2002) que permite resolver dificultades y sobreponerse a ellas, mediante el apoyo de terceros, movilizando una serie de competencias de naturaleza cognitivas, sociales y emocionales (Fatoux et al., 2018; Finney et al., 2018).

En términos generales, respecto de este proceso, se ha distinguido entre búsqueda de ayuda instrumental y ejecutiva (Karabenick y Gonida, 2018; Nelson-Le Gall, 1981). Sin embargo, a lo largo de estos años, la investigación indica que la comprensión del fenómeno de búsqueda de ayuda posee diversas dimensiones y complejidades (Karabenick y Dembo, 2011). Para una visión general y detallada, ver Bornschlegl et al. (2020), Chowdhury and Halder (2019), Karabenick and Berger (2013).

La búsqueda de esta ayuda académica puede concretarse a través de distintas estrategias: a través de mecanismos informales o formales. En general, ambos mecanismos tienen potenciales beneficios y dificultades (Heerde y Hemphill, 2018; Jaroszewicz, 2020). La búsqueda informal se concreta fundamentalmente con pares cercanos o solicitando directamente esta ayuda a profesores o ayudantes de cátedra. No obstante, muchas veces, las necesidades de ayuda exceden lo que estos agentes pueden, de manera eficaz, apoyar. En este contexto las instituciones han diseñado instancias de apoyo académico de diverso tipo.

Sin embargo, diversos reportes muestran que los estudiantes que más lo necesitan, no acceden a estos apoyos, al menos de manera oportuna (Finney et al., 2018). En muchos casos, la cercanía es el criterio que opera para optar por la vía informal de búsqueda de ayuda y no por los apoyos institucionales, es decir, por aquella ayuda más próxima o segura; en desmedro 
de la experticia para brindar el apoyo específico requerido (Amemiya y Wang, 2017; Kiefer y Shim, 2016; Zander et al., 2019).

Las razones que explicarían esta disposición a participar de los apoyos institucionales, como variable mediadora de la participación efectiva en los AAI, son múltiples, y van desde la vergüenza y la estigmatización que puede significar la participación en estos apoyos hasta cuestiones prácticas o logísticas (por ejemplo, los AAI se ofertan en un campus distinto al que pertenece el estudiante).

A lo anterior hay que añadir, la valoración que los estudiantes tienen de estas instancias de apoyo ofertadas por sus instituciones (McCabe, 2018). Así, la disposición general de los estudiantes a recurrir a los apoyos que brinda la institución en caso de tener dificultades académicas, aparece como una variable clave que facilitaría la concreción de recurrir efectivamente a estos AAI.

En el proceso de búsqueda de ayuda es posible establecer un conjunto de pasos o etapas que pueden ser ejecutadas por los estudiantes (ver figura 1). Sin embargo, este proceso no se presenta necesariamente de manera secuencial en todos los sujetos, aunque siempre inicia con el reconocimiento por parte del estudiante de que existe un problema y que debe ser resuelto (Reeves, 2014).

La resolución de un problema de esta índole requiere que el estudiante evalúe los costos y beneficios asociados a la demanda de ayuda de tipo formal o informal (Wirtz et al., 2018). Esto último, en el contexto de este estudio, es relevante ya que las instituciones de enseñanza superior proveen de manera cada vez más extendida apoyos académicos centralizados, para sus estudiantes (Osorio Vargas, 2019; Venegas y Gairín, 2020).

Si bien la búsqueda de ayuda ha sido un tópico bastante investigado, se sabe poco respecto de las variables que podrían predecir la participación de los estudiantes en este tipo de dispositivos (Collins y Sims, 2006; Means y Pyne, 2017). 
Figura 1. Proceso de búsqueda de ayuda académica (Nelson-Le Gall, 1981; Karabenic, 2014).

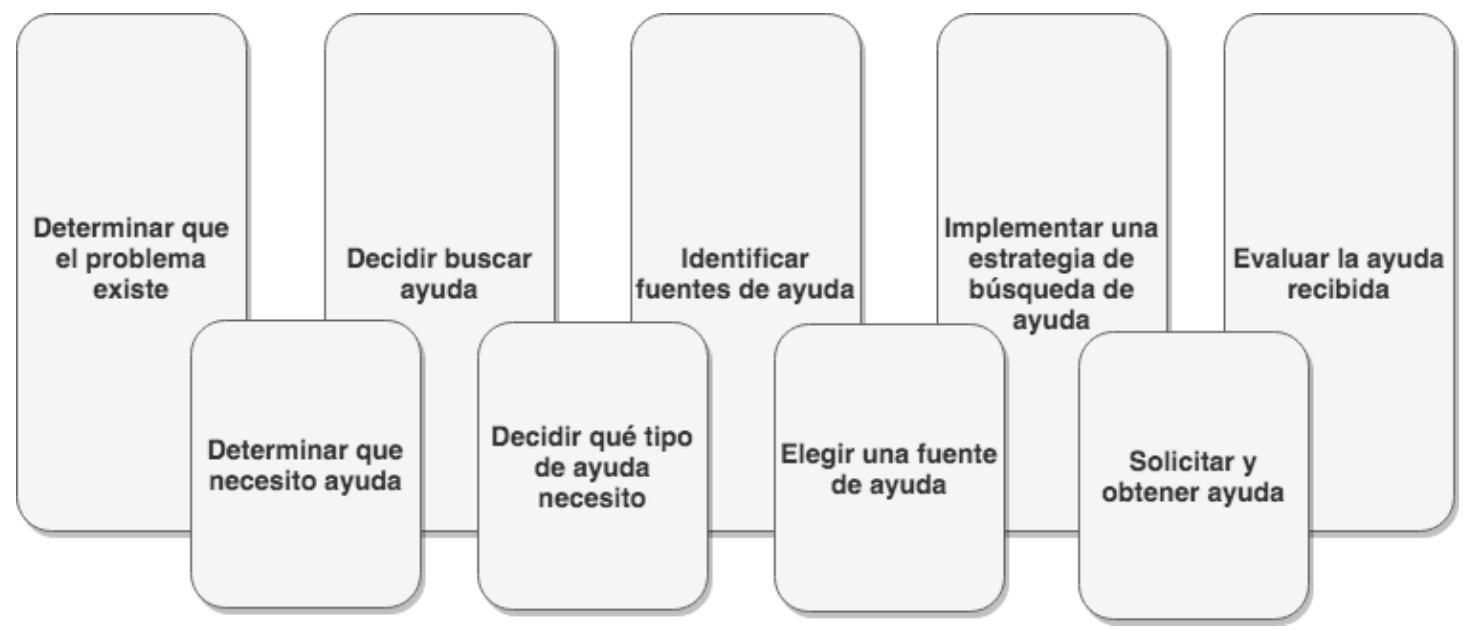

Fuente: Reeves (2014).

\section{La participación en dispositivos AAI}

Abordar la cuestión de la participación en los AAI es complejo dado que no todas las instituciones se hacen cargo de los mismos tipos de necesidades, y aun haciéndolo, no ofertan los mismos tipos de apoyo. Esto depende de las capacidades institucionales, en el ámbito profesional y financiero, para sostener iniciativas más o menos amplias de apoyos académicos. Por otra parte, las necesidades de los estudiantes no son siempre las mismas y abarcan una amplia gama de apoyos posibles (Valenzuela et al., 2021).

Por lo anterior, para este estudio, se ha identificado una serie de variables que la literatura asocia al proceso de búsqueda de ayuda que, junto a otras variables, propias del contexto institucional, podrían ayudar a comprender la participación en los dispositivos de apoyo académico que las instituciones ofertan. Como una forma de organizar estas variables, se han agrupado en dos grupos: variables personales (conciencia de dificultad, experiencias previas, variables motivacionales y variables inhibitorias) $\mathrm{y}$, por otra parte, variables condicionantes vinculadas a los AAI (conocimiento de la oferta e identificación de la pertinencia).

\section{Variables Personales}

Dentro de esta categoría de variables se agruparon aquellas asociadas al individuo y que la literatura ha reportado como factores que inciden en la búsqueda de ayuda, siendo 
estas: la conciencia de la dificultad, la conciencia de la necesidad de apoyo, la experiencia previa de búsqueda, el compromiso académico, la vergüenza y el costo de la actividad.

En el proceso de búsqueda de ayuda, la primera variable que emerge como condición personal fundamental es la conciencia del individuo de la dificultad que se está experimentando. Tener conciencia de la dificultad (en este caso, académica) es una condición necesaria para iniciar este proceso. No obstante, ello no es condición suficiente para que esa dificultad sea subsanada a través de la búsqueda de apoyo. El proceso de búsqueda de ayuda requiere además la conciencia de que se requiere ayuda o apoyos de un tercero. En los diversos modelos de búsqueda de ayuda, ya sea de manera implícita como en Nelson-Le Gall (1981) o explícita en Karabenick (2014) o Reeves (2014), este primer paso dentro del proceso de búsqueda de ayuda está mediado por la conciencia de tener una dificultad que requiere apoyo para su resolución.

Por su parte, la literatura reporta que la experiencia previa de búsqueda exitosa de ayuda es una variable relevante para facilitar nuevos eventos de búsqueda de ayuda (Çebi y Demir, 2020; Jaroszewicz, 2020). El haber recibido apoyos en etapas previas (por ejemplo, en la educación secundaria) podría contribuir a reducir un eventual sentimiento de vergüenza o el estigma de requerir apoyos, cuando otros no lo necesitan. En este sentido, la experiencia exitosa anterior de haber dado el paso de buscar ayuda con otros agentes (no institucionales) podría disponer al estudiante a explorar la vía institucional como alternativa de solución de las dificultades académicas que enfrenta.

Por otra parte, en el plano motivacional (Eccles y Wigfield, 2020; Wigfield et al., 2016), el proceso de búsqueda de ayuda se potenciaría en la medida que solucionar las dificultades académicas sea evaluado como importante por el sujeto (Amemiya y Wang, 2017). Esta disposición motivacional se traduce a su vez en compromiso académico (Martin et al., 2017). Este compromiso facilitaría, en caso de dificultades académicas, el buscar ayuda (Duchesne et al., 2019). En este contexto la investigación motivacional ha reportado una relación virtuosa entre las percepciones de competencia de los estudiantes y sus creencias de autoeficacia, así como las metas de logro centradas en el aprendizaje y la búsqueda de ayuda (Gonida et al., 2019; Hao et al., 2017; Won et al., 2019). 
Pero no todas las variables tienen un efecto positivo sobre la búsqueda de ayuda. Hay variables que actúan en sentido inverso, generando un efecto inhibitorio. Estas variables inhibitorias generarían una dinámica de evitación respecto a esta estrategia para acceder a ayudas académicas. Dentro de este ámbito se destacan dos variables fundamentales que actúan como inhibidores de la conducta en la búsqueda de ayuda en general y que eventualmente mantienen un efecto significativo en cuando esta búsqueda se canaliza a través de los dispositivos institucionales: la vergüenza y el costo de la actividad. La vergüenza, en tanto emoción negativa (Pekrun et al., 2006), tendría un efecto inhibitorio en la concreción de la posibilidad de pedir ayuda (Apto et al., 2017; Hartman, 2019). Esto, eventualmente, sería especialmente importante en contextos socialmente más amplios tales como la sala de clase o situaciones que implican una exposición social mayor (Sanchez-Rosas et al., 2016). En este sentido, y a falta de evidencia al respecto, sólo se puede hipotetizar que esta variable podría tener un efecto significativo para explicar la participación en dispositivos de ayuda académica a nivel institucional.

Por su parte, se constata que en la medida que mayor sea el costo de la actividad, en este caso de pedir ayuda a nivel institucional, menores serán las probabilidades de que esta necesidad se concrete bajo esta modalidad. Sin embargo, el costo no es una variable unidimensional. Flake et al. (2015) distinguen al menos cuatro tipos de costo, aportando de esta manera distinciones importantes para mirar el fenómeno de manera más comprensiva. Para estos autores, es posible identificar cuatro tipos de costo: el costo de la tarea (Task Effort Cost) esto es el trabajo o esfuerzo implicados en la realización de una actividad, el costo que significa dejar de hacer otras cosas que son consideradas valiosas (LOVA, Loss of Valued Alternatives); un tercer tipo de costo es aquel que surge de realizar una actividad que no es deseada o no es de interés y finalmente, el costo emocional, entendido como el estado emocional negativo que resulta de realizar una actividad que no es de mi interés.

\section{Variables condicionantes vinculadas a los AAI}

En el contexto de la búsqueda de apoyo en las instituciones es necesario considerar, al menos, dos variables específicas que condicionan el que este tipo específico de ayuda sea vista como una alternativa válida por el estudiante. En primer lugar, el conocimiento de la existencia de estos dispositivos institucionales. Evidentemente, si se desconoce la existencia de estos apoyos, es muy difícil que un estudiante oriente su búsqueda de ayuda hacia dispositivos que no están en su horizonte. No obstante ello, e incluso sabiendo que existen 
estos apoyos institucionales, es necesario que los mecanismos específicos de acceso a ellos sean conocidos. Lo anterior implica, además, identificar de manera concreta los requisitos y pasos que se deben cumplir para concretar la demanda (Reeves, 2014). Por ejemplo, reconocer la oficina a la que se debe dirigir, o identificar a la persona a quien se puede recurrir para concretar la solicitud de ayuda, ya sea a nivel presencial o virtual.

Sin embargo, esta condición por sí misma no pareciera ser suficiente. En efecto, para que se concrete la demanda de ayuda, el conocimiento de la existencia de apoyos institucionales debe interactuar con la percepción de pertinencia para sí mismos que los estudiantes atribuyen a estos apoyos académicos institucionales. De nada sirve un conocimiento procedimental de acceso a estos dispositivos, si los AAI no son vistos como una oferta de apoyo adecuada y pertinente para el tipo de necesidad que el estudiante que la busca requiere (identificación).

A la identificación de la pertinencia de los dispositivos se debe añadir la valoración que los estudiantes realizan de dichos apoyos. De esta manera, la percepción que se tiene de los AAI que oferta la institución y el conocimiento de los procedimientos para acceder a ellos constituyen factores que aportarían al sujeto la disposición para concretar el uso y aprovechamiento de las iniciativas de apoyo definidas institucionalmente.

\section{Objetivo}

El objetivo de este estudio fue evaluar la capacidad predictiva de un conjunto de variables sobre la probabilidad de ocurrencia de la participación de los estudiantes en dispositivos de apoyo académicos institucionales (AAI). Ello permitirá, por una parte, constatar en qué medida las variables predictoras de la búsqueda de ayuda, en general, son también predictoras en el caso de que esta ayuda se realice en un marco institucional, y por otra, dar pistas a las instituciones que contribuyan a facilitar el acceso a estos dispositivos a los estudiantes que lo requieren.

\section{Método}

\section{Participantes}

Este estudio se enmarca dentro de un proyecto mayor que indagó en la participación en AAI en universidades chilenas (Fondecyt 1181159). En este estudio participaron 
estudiantes universitarios mayores de 18 años, de ambos sexos, que cursaban carreras de pregrado en 10 áreas de conocimiento en 4 universidades chilenas (ver Tabla 1). Estas universidades corresponden a instituciones públicas y privadas, con 4 y 5 años de acreditación. Esto es, un tipo de universidad con rango intermedio de acreditación institucional y que atiende al $57.7 \%$ del estudiantado universitario chileno (Muñoz y Blanco, 2013).

En consideración de que el objetivo de esta investigación es evaluar los factores que inciden en la participación en AAI, para el análisis de datos se excluyeron aquellos casos en los cuales los estudiantes declararon haber participado en apoyos institucionales de manera obligatoria ( $n=50)$. Así la muestra final estuvo constituida por 803 casos $(71,1 \%$ Mujeres) con edad promedio de 22.81años $(S D=4,81)$.

Tabla 1. Participantes por área de conocimiento y sexo

\begin{tabular}{lrccc}
\hline Área de conocimiento & Frec. & $\%$ & Varones & Mujeres \\
\hline Administración y Comercio & 71 & 8.8 & $36.6 \%$ & $63.4 \%$ \\
Agropecuaria & 45 & 5.6 & $33.3 \%$ & $66.7 \%$ \\
Arte y Arquitectura & 11 & 1.4 & $9.1 \%$ & $90.9 \%$ \\
Ciencias Básicas & 25 & 3.1 & $40.0 \%$ & $60.0 \%$ \\
Ciencias Sociales & 123 & 15.3 & $23.6 \%$ & $76.4 \%$ \\
Derecho & 52 & 6.5 & $26.9 \%$ & $73.1 \%$ \\
Educación & 229 & 28.5 & $22.7 \%$ & $77.3 \%$ \\
Humanidades & 11 & 1.5 & $18.2 \%$ & $81.8 \%$ \\
Salud & 155 & 19.3 & $21.3 \%$ & $78.7 \%$ \\
Tecnología & 81 & 10.1 & $61.7 \%$ & $38.3 \%$ \\
\hline Total & 803 & 100 & $28.9 \%$ & $71.1 \%$ \\
\hline
\end{tabular}

\section{Instrumentos}

Para dar cuenta de las distintas variables de interés se elaboró un cuestionario para ser auto aplicado en modalidad online. Este cuestionario consideró la operacionalización de la variable dependiente Participación en AAI (BAAI) y catorce variables independientes (ver Tablas 2 y 3). La operacionalización de variables consideró ítems con alternativas de respuesta dicotómicas y otros con alternativas politómicas tipo Likert.

Las variables observadas medidas a través de reactivos dicotómicos (ver Tabla 2), corresponden a las variables dependiente Participación en los Apoyos Institucionales (BAAI), y a seis variables independientes dicotómicas: Conciencia de dificultad académica $(C D)$, 
Necesidad de apoyos académicos (NAA); Búsqueda previa de Ayuda (BAA); Conocimiento de la existencia de AAI (AAI conocimiento); Identificación como destinatario de los AAI ofertados por la institución (AAI identificación) y, Conocimiento de los mecanismos de acceso a los AAI ofertados por la institución (AAI acceso). Estas variables se construyeron de esta manera debido a que la naturaleza de la información requerida no se relaciona con constructos latentes o complejos, y por lo tanto podía ser eficazmente abordada a través de ítemes dicotómicos (del tipo "participa o no en este tipo de actividades"). Estos reactivos fueron validados por un grupo de jueces (doctores en educación y especialistas en el área de apoyos académicos), quienes se pronunciaron sobre su validez de contenido y la pertinencia de incorporarlas dentro del diseño.

Tabla 2. Variables dicotómicas (Sí-No)

\begin{tabular}{|c|c|c|}
\hline Etiqueta & Variable & Ítems \\
\hline BAAI & $\begin{array}{l}\text { Participación en } \\
\text { AAI }\end{array}$ & $\begin{array}{l}\text { "He participado en alguna actividad de apoyo académico ofrecida } \\
\text { por mi institución" }\end{array}$ \\
\hline $\mathrm{CD}$ & $\begin{array}{l}\text { Conciencia de } \\
\text { dificultad } \\
\text { académica. }\end{array}$ & $\begin{array}{l}\text { "Durante mis estudios en esta institución ¿he tenido dificultades } \\
\text { académicas? (problemas para aprender o para tener un } \\
\text { rendimiento adecuado)" }\end{array}$ \\
\hline NAA & $\begin{array}{l}\text { Necesidad de } \\
\text { apoyos académicos. }\end{array}$ & $\begin{array}{l}\text { "Durante mis estudios en esta institución ¿he necesitado ayuda de } \\
\text { otros para aprender o para mejorar mi rendimiento?" }\end{array}$ \\
\hline BAA & $\begin{array}{l}\text { Búsqueda previa de } \\
\text { ayuda. }\end{array}$ & "Durante mi carrera ¿he buscado ayudas en el plano académico" \\
\hline $\begin{array}{l}\text { AAI } \\
\text { Conocimiento }\end{array}$ & $\begin{array}{l}\text { Conocimiento de la } \\
\text { existencia de AAI. }\end{array}$ & $\begin{array}{l}\text { "Mi institución ofrece apoyos académicos (ej. Talleres, cursos, } \\
\text { atención psicopedagógica, etc.) }\end{array}$ \\
\hline $\begin{array}{l}\text { AAI } \\
\text { Identificación }\end{array}$ & $\begin{array}{l}\text { Identificación como } \\
\text { destinatario de los } \\
\text { AAI. }\end{array}$ & $\begin{array}{l}\text { "Los apoyos académicos de mi institución ¿están dirigidos a } \\
\text { estudiantes como yo?" }\end{array}$ \\
\hline $\begin{array}{l}\text { AAI } \\
\text { Acceso }\end{array}$ & $\begin{array}{l}\text { Conocimiento de } \\
\text { acceso a AAI. }\end{array}$ & $\begin{array}{l}\text { "¿Sabría cómo acceder o benefíciarme de esos apoyos en caso de } \\
\text { necesitarlos? (ej. Talleres, cursos, atención psicopedagógica, } \\
\text { etc.)" }\end{array}$ \\
\hline
\end{tabular}

De igual manera, se recurrió a variables que corresponden a constructos latentes y que requieren medición a través de escalas. Estas variables se midieron a través de ítems policotómicos tipo Likert de 6 puntos (1= muy en desacuerdo, $6=$ muy de acuerdo). Con la excepción de la variable Disposición a buscar ayuda académica a través de AAI (Disposición), el resto de las variables consideraron el cálculo de escalas unifactoriales (Ver Tabla 3).

La Valoración atribuida a los AAI de la propia institución (AAI valoración), se midió a través de una escala unifactorial de 4 ítems tipo Likert de 6 puntos $(1=$ muy en desacuerdo - 
$6=$ muy de acuerdo). La escala muestra buenos índices de ajuste $\left.\chi_{(2)}^{2}\right)=.924, p=.639$, $\mathrm{RMSEA}=.00[.00-.006], \mathrm{SRMR}=.00, \mathrm{TLI}=1, \mathrm{CFI}=1$ y una alta confiabilidad $(\omega$ de McDonald >.827.).

Tabla 3. Variables Tipo Likert de 6 puntos $(1=$ muy en desacuerdo, $6=$ muy de acuerdo $)$

\begin{tabular}{|c|c|c|}
\hline Etiqueta & Variable & Ítems \\
\hline \multirow{4}{*}{$\begin{array}{l}\text { Valoración } \\
\text { AAI }\end{array}$} & \multirow{4}{*}{$\begin{array}{l}\text { Valoración } \\
\text { atribuida a } \\
\text { los AAI de la } \\
\text { propia } \\
\text { institución }\end{array}$} & Los Apoyos Académicos de mi institución son valiosos \\
\hline & & Los Apoyos Académicos de mi institución son útiles \\
\hline & & Los Apoyos Académicos de mi institución son necesarios \\
\hline & & En realidad, los Apoyos Académicos de mi institución no son de mucha ayuda \\
\hline \multirow{4}{*}{ Engagement } & \multirow{4}{*}{$\begin{array}{l}\text { Compromiso } \\
\text { académico. }\end{array}$} & Invierto tiempo y esfuerzo en alcanzar un aprendizaje de calidad \\
\hline & & $\begin{array}{l}\text { En mi estudio, dedico todo el tiempo necesario para alcanzar un dominio total del } \\
\text { contenido }\end{array}$ \\
\hline & & $\begin{array}{l}\text { Si mi dominio de la materia no es suficiente, soy capaz de invertir más tiempo en } \\
\text { lograr un aprendizaje de calidad }\end{array}$ \\
\hline & & Me doy tiempo para profundizar en las materias que me enseñan en la carrera. \\
\hline $\begin{array}{l}\text { Disposición } \\
\text { AAI }\end{array}$ & $\begin{array}{l}\text { Disposición a } \\
\text { buscar ayuda } \\
\text { académica a } \\
\text { través de } \\
\text { AAI. }\end{array}$ & En caso de tener dificultades recurriría a los apoyos que ofrece mi institución. \\
\hline \multirow{4}{*}{ TC } & \multirow{4}{*}{$\begin{array}{l}\text { Task Effort } \\
\text { Cost }\end{array}$} & $\begin{array}{l}\text { Pedir ayuda en los apoyos que brinda mi institución demandan tiempo que no } \\
\text { tengo }\end{array}$ \\
\hline & & Pedir ayuda en los apoyos que brinda mi institución implica mucho trabajo \\
\hline & & Pedir ayuda en los apoyos que brinda mi institución implica demasiado esfuerzo \\
\hline & & Pedir ayuda en los apoyos que brinda mi institución demanda mucha energía \\
\hline \multirow{4}{*}{ LOVA } & \multirow{4}{*}{$\begin{array}{l}\text { Loss of } \\
\text { Valued } \\
\text { Alternatives }\end{array}$} & $\begin{array}{l}\text { No importa cuánto trabajo tenga siempre hago un esfuerzo por aprovechar los } \\
\text { apoyos de mi institución }\end{array}$ \\
\hline & & Me exige renunciar a otras actividades que valoro \\
\hline & & Implica renunciar a otras actividades que me importan \\
\hline & & Me quita un tiempo valioso para hacer otras cosas \\
\hline \multirow{4}{*}{ OEC } & \multirow{4}{*}{$\begin{array}{l}\text { Outside } \\
\text { Effort Cost, } \\
\text { OEC }\end{array}$} & Implica que tengo que dejar de lado demasiadas cosas \\
\hline & & $\begin{array}{l}\text { No es posible, ya que debido a otras demandas, no tengo tiempo suficiente para } \\
\text { asistir }\end{array}$ \\
\hline & & Es muy complicado ya que tengo otras responsabilidades \\
\hline & & Por otras cosas que hago, no tengo tiempo para ir \\
\hline \multirow{4}{*}{$\mathrm{EC}$} & \multirow{4}{*}{$\begin{array}{l}\text { Emotional } \\
\text { Cost }\end{array}$} & Es emocionalmente agotador \\
\hline & & Es muy frustrante \\
\hline & & Es muy estresante \\
\hline & & Me hace sentir muy ansioso \\
\hline \multirow{4}{*}{ Vergüenza } & \multirow{4}{*}{ Vergüenza } & Me da mucha vergüenza que en mi institución sepan que necesito ayuda \\
\hline & & Soy muy orgulloso(a) como para pedir ayuda \\
\hline & & Pedir ayuda a otros me hace sentir tonto \\
\hline & & Pedir ayuda a otros me hace sentir menos que mis compañeros \\
\hline
\end{tabular}


El Compromiso académico (Engagement), a través de una escala unidimensional de 4 ítems, con similares características que la anterior, muestra validez $\chi_{(2)}^{2}=2.17, p=.34$, $\mathrm{RMSEA}=.01$ [.00-.082], SRMR $=.01, \mathrm{TLI}=1, \mathrm{CFI}=1 \mathrm{y}$ un buen índice de confiabilidad $\omega \mathrm{de}$ McDonald >.867.

La Disposición a buscar ayuda académica a través de AAI (Disposición) se evaluó a través una pregunta tipo Likert de 6 puntos en donde se preguntaba a los estudiantes por su grado de acuerdo con la afirmación: "En caso de tener dificultades recurriría a los apoyos que ofrece mi institución".

El costo de la tarea de participar de AAI fue evaluada a través de una escala ad-hoc inspirada en Flake et al., (2015). Corresponde también a una escala tipo Likert de 6 puntos que evalúa cuatro subdimensiones: el costo de la tarea (Task Effort Cost, TC), la pérdida de alternativa valiosa (Loss of Valued Alternatives, LOVA), el costo externo (Outside Effort Cost, OEC) el que corresponde al costo de hacer algo que no es de interés y el costo emocional (Emotional Cost, EC). La escala evidencia validez $\chi_{(57)}^{2}=1.85 ; p<.01$; RMSEA= .07 [.060-.078], SRMR= .04, CFI= .97, TLI $=.97 \mathrm{y}$ alta confiabilidad en todas las subescalas $(\omega$ McDonald > .834).

Finalmente, la vergüenza respecto a solicitar ayuda fue evaluada a través de una escala de 4 ítems tipo Likert, en una escala de 6 puntos cuyos índices de ajuste dan cuenta de su validez $\chi_{(2)}^{2}=.01 ; p<.93 ; \mathrm{RMSEA}=.00$ [.00-.05], SRMR<.01, CFI=1, TLI=1; y cuyo nivel de confiabilidad fue de $\omega$ McDonald $=.846$.

\section{Procedimiento}

La toma de datos se realizó de manera on-line con el apoyo de las cuatro universidades participantes, quienes enviaron una invitación a los estudiantes de pregrado a través de correo electrónico. La invitación contenía un link a la página de presentación del estudio y al consentimiento informado.

El texto del consentimiento informado fue aprobado previamente por un comité ético científico acreditado. Tanto el consentimiento informado como la declaración de mayoría de edad de los participantes fueron considerados como filtros para participar del estudio. Se 
dejaba además constancia del compromiso de confidencialidad de los datos, por parte del equipo de investigación. El instrumento recogía además datos sociodemográficos entre los cuales estaba la edad. En el caso que los estudiantes declararan tener menos de 18 años (edad en la que se adquiere la mayoría de edad en Chile), el sistema impedía contestar el instrumento. En dicho consentimiento el estudiante accedía a participar de manera libre y voluntaria del estudio. Solo una vez aceptados los términos de participación, se abría el acceso al cuestionario on-line.

La recolección de datos se realizó durante el segundo semestre de 2019. En este período se logró una muestra total de 853 casos. De esta muestra total se seleccionó una submuestra de la cual se excluyó a aquellos estudiantes que manifestaron haber participado en apoyos académicos institucionales de manera no voluntaria. Así, los casos de participación obligatoria $(n=50)$ fueron excluidos y la muestra efectiva corresponde a 803 casos.

\section{Análisis de datos}

Para evaluar la capacidad predictiva de las variables del estudio en la probabilidad de ocurrencia de la participación de los estudiantes en estos dispositivos se realizó un análisis de regresión logística binomial con asistencia del software Jamovi (The Jamovi Proyect, 2019).

La regresión logística binaria múltiple es uno de los métodos más utilizados entre los modelos lineales generalizados (MLG). Dichos modelos extienden los procedimientos de regresión lineal a situaciones en que no se cumplen algunos de los supuestos de ésta. En particular, la regresión logística, permite evaluar la capacidad predictiva de un conjunto de variables sobre una variable dependiente dicotómica (Fox, 2016). En este caso, se hace sobre la variable participación en sistemas de apoyos académicos que ofertan las instituciones universitarias (AAI), en donde la participación en dichos apoyos ha sido codificada como 1 y la no participación como 0 .

Como es sabido, este método no modela directamente la variable dependiente medida de manera dicotómica. Considera en cambio, la transformación de la variable dependiente en un logaritmo de odds, es decir, el logaritmo de la razón entre la probabilidad de ocurrencia del evento sobre la probabilidad de no ocurrencia (Cerda et al., 2013). Si bien los odds son solo una manera particular de expresar probabilidades, en el contexto hispano americano son expresiones poco utilizadas (Cea D'ancona, 2004; Silva y Barroso, 2004), y es razonable 
comentarlas, especialmente para el caso de las consecuencias sobre los valores de los coeficientes usualmente interpretados en el marco del análisis de regresión.

En este contexto, debe considerarse que las magnitudes de los coeficientes del modelo no pueden interpretarse directamente como es usual en la regresión lineal, ya que están expresados como logaritmos naturales de odds (columna "Estimate"). Se interpretarán en cambio las respectivas transformaciones exponenciales de dichos coeficientes expresadas como "Odds" (columna "Odds ratio"). Estos últimos coeficientes transformados son los que resultan claves en la interpretación del modelo ajustado. En el caso de las variables independientes, los valores superiores a 1 de los mismos, implicarán un aumento en la probabilidad de participación en AAI, manteniéndose el resto de las variables inalteradas (cf. Persoskie y Ferrer, 2017).

\section{Resultados}

\section{Análisis descriptivos}

Los resultados en las variables observadas y medidas a través de reactivos dicotómicos (sí-no) mostraron una alta tasa de respuestas afirmativas en todos los casos, con la sola excepción de la variable participación en dispositivos de apoyos institucionales (BAAI), no evidenciándose diferencias por sexo en ninguna de ellas (ver Tabla 4).

Tabla 4. Variables dicotómicas. Porcentajes de Sí, para muestra general y por sexo *

\begin{tabular}{lccc}
\hline & Muestra General & Mujeres & Varones \\
\hline CD & 64.2 & 64.9 & 62.2 \\
NAA & 78.1 & 79.3 & 75.1 \\
BAA & 67,0 & 66.9 & 68.4 \\
AAI Identificación & 82.9 & 84.2 & 80.0 \\
AAI Conocimiento & 77.5 & 76.8 & 79.6 \\
AAI Acceso & 67.8 & 65.0 & 73.7 \\
BAAI & 47.5 & 46.9 & 49.4 \\
\hline CD= Conciencia de dificultad; NAA= Necesidad de Apoyos académicos; BAA= Búsqueda de ayuda (general); AAI \\
identificación= identifica a los apoyos como destinados a estudiantes como el/ella; AAI conocimiento= Conocimiento de la \\
existencia de Apoyos institucionales; AAI acceso= Conocimiento de mecanismos de acceso a los Apoyos institucionales; \\
BAAI= Búsqueda de apoyos a nivel institucional. \\
* Nota: no se evidencian diferencias significativas entre los porcentajes entre mujeres y hombres $(p>.05)$
\end{tabular}


Por su parte, los constructos latentes que fueron medidos en escalas de 1 a 6 muestran medias superiores al punto medio de la escala en los casos de las variables valoración, engagement y disposición a buscar ayuda en los dispositivos institucionales, mientras que las variables de tipo inhibitorio mostraron un comportamiento opuesto, mostrando así bajos niveles de costo y vergüenza (ver Tabla 5). En este caso se observaron diferencias en el tipo de costo OEC y EC, donde las mujeres declaran que participar de los AAI tiene un mayor costo como actividad que no es de interés $(p=.012)$ y costo emocional $(p=.018)$ que sus pares varones.

Tabla 5. Descriptivos de variables continuas por sexo

\begin{tabular}{lcccccc}
\hline & \multicolumn{2}{c}{ Muestra } & \multicolumn{2}{c}{ Mujeres } & \multicolumn{2}{c}{ Varones } \\
& $M$ & $S D$ & $M$ & $S D$ & $M$ & $S D$. \\
\hline Valoración de AAI & 4.65 & 1.02 & 4.65 & 1.00 & 4.65 & 1.07 \\
Engagement & 4.54 & 0.98 & 4.57 & 0.94 & 4.46 & 1.06 \\
Disposición AAI & 4.09 & 1.44 & 4.12 & 1.43 & 4.02 & 1.48 \\
Task Effort Cost(TC) & 2.97 & 1.24 & 3.01 & 1.26 & 2.86 & 1.18 \\
Outside Effort Cost (OEC) & 3.64 & 1.50 & 3.75 & 1.53 & 3.40 & 1.41 \\
Loss of Valued Alternatives (LOVA) & 3.53 & 1.23 & 3.54 & 1.25 & 3.51 & 1.17 \\
Emotional Cost (EC) & 2.68 & 1.29 & 2.76 & 1.33 & 2.47 & 1.17 \\
Vergüienza & 2.21 & 1.25 & 2.18 & 1.22 & 2.28 & 1.33 \\
\hline
\end{tabular}

Regresión logística

El análisis de regresión logística realizado mostró la pertinencia de 6 de las 14 variables consideradas inicialmente en el análisis. Las variables excluidas del análisis final fueron: el conocimiento de la existencia de apoyos académicos institucionales ofertados por la universidad; la identificación del estudiantes como destinatario de esos AAI; la valoración que los estudiantes realizan de los AAI ofertados por la institución; el compromiso académico general (engagement); la vergüenza y los tipos de costos asociados con el esfuerzo implicado en la tarea (TC), el esfuerzo de realizar una actividad que no es de interés (outside effort cost) y el costo emocional (EC). En todos estos casos el aporte predictivo no fue significativo ( $p>$ $.05)$.

Así, las variables retenidas finalmente (ver Tabla 6) fueron: la Búsqueda de ayuda general; la conciencia de dificultad académica, la conciencia de necesidad de ayuda, el conocimiento de los procedimientos concretos para acceder a los AAI, la disposición a 
buscar ayuda a nivel institucional, y finalmente, la dimensión del costo relacionada con la pérdida de alternativas valiosas (LOVA).

Tabla 6. Coeficientes retenidos en el modelo de regresión logística (BAAI)

\begin{tabular}{|c|c|c|c|c|c|c|c|c|c|}
\hline \multirow[b]{2}{*}{ Predictor } & \multirow[b]{2}{*}{$B$} & \multicolumn{2}{|l|}{$95 \% \mathrm{CI}$} & \multirow[b]{2}{*}{$S E$} & \multirow[b]{2}{*}{$Z$} & \multirow[b]{2}{*}{$p$} & \multirow[b]{2}{*}{ Odds ratio } & \multicolumn{2}{|l|}{$95 \% \mathrm{CI}$} \\
\hline & & Lower & Upper & & & & & Lower & Upper \\
\hline Intercept & -2.68 & -3.87 & -1.49 & 0.61 & -4.42 & $<.001$ & 0.07 & 0.02 & 0.22 \\
\hline $\begin{array}{l}\text { BAA: } \\
(\mathrm{Sí}-\mathrm{No})\end{array}$ & 1.45 & 0.98 & 1.91 & 0.24 & 6.12 & $<.001$ & 4.25 & 2.67 & 6.75 \\
\hline $\begin{array}{l}\text { AAI acceso: } \\
\text { (Sí - No) }\end{array}$ & 1.25 & 0.77 & 1.72 & 0.24 & 5.15 & $<.001$ & 3.48 & 2.17 & 5.60 \\
\hline $\begin{array}{l}\text { NAA: } \\
\text { (Sí - No) }\end{array}$ & 0.81 & 0.27 & 1.36 & 0.28 & 2.93 & .003 & 2.25 & 1.31 & 3.88 \\
\hline $\begin{array}{l}\text { CD: } \\
(\mathrm{Sí}-\mathrm{No})\end{array}$ & 0.59 & 0.11 & 1.06 & 0.24 & 2.42 & .015 & 1.80 & 1.12 & 2.88 \\
\hline Disposición AAI & 0.24 & 0.07 & 0.40 & 0.08 & 2.80 & .005 & 1.27 & 1.07 & 1.50 \\
\hline LOVA & -0.34 & -0.51 & -0.16 & 0.09 & -3.76 & $<.001$ & 0.71 & 0.60 & 0.85 \\
\hline
\end{tabular}

Nota. Estimates (B) representan el $\log$ odds de "BAAI = participa" vs. "BAAI = No participa". BAA= Búsqueda de ayuda (general); AAI acceso= Conocimiento de mecanismos de acceso a los Apoyos institucionales; $\mathrm{NAA}=$ Necesidad de Apoyos académicos; $\mathrm{CD}=$ Conciencia de dificultad; LOVA $=$ Loss of valuable alternatives

El modelo muestra una devianza positiva que da cuenta de un efecto significativo del modelo propuesto $\left(\chi_{(6)}^{2}=170 ; p<.001\right)$. El modelo resultante predice una porción significativa de la varianza que oscila entre un pseudo $R^{2}$ de Mc Fadden $=.237$ y un $R^{2}$ de Nagelkerke $=.373$, prediciendo correctamente un $74.75 \%$ de los casos, más específicamente, un $75.7 \%$ de los casos que no participan de los AAI y un $73.8 \%$ de aquellos casos que concretaron su participación en algún dispositivo de apoyo académico ofertado por la institución.

\section{Análisis de las variables predictoras}

Búsqueda de ayuda académica (BAA). Los resultados muestran que los estudiantes que han buscado ayuda en general (amigos, pares, familia, otros) tienen 4.25 veces más probabilidades de participar de los AAI que los que no buscaron dicha ayuda.

Lo anterior implica que, en promedio, existe una probabilidad de un $82.4 \%$ de que un estudiante participe en AAI, habiendo buscado. 
Conciencia de dificultad. La conciencia de dificultad académica, como primer paso del proceso de búsqueda de ayuda contribuye a la predicción de la participación con un Odds Ratio de 1.80 esto es, que aquellos estudiantes con conciencia de dificultades académicas tiene 1,8 veces más probabilidades de participar de los apoyos institucionales que aquellos que no la tienen, o dicho de otro modo, que en promedio existe una probabilidad de $71.35 \%$ de que un estudiante participe en AAI cuando tiene conciencia de su dificultad respecto a no tener dicha conciencia. Así, la probabilidad de que los estudiantes finalmente participen de los AAI es de .246 en el caso de los que no tienen conciencia de dificultades académicas y de .37 en quienes sí la tienen.

Acceso a los AAI. El conocimiento preciso de cómo acceder a los dispositivos de apoyo académico institucional, esto es conocer dónde inscribirse, con quien gestionar su participación, o conocer la oficina o el encargado de realizar los procedimientos para acceder a los AAI aparece como una variable relevante. Los estudiantes que saben cómo acceder a los AAI ofertados por la institución tienen 3.48 veces más posibilidades de acceder a ellos que sus compañeros que desconocen el procedimiento.

De esta manera, hay una probabilidad de un $78 \%$ que una persona que conozca los procedimientos participe en los AAI, que si no lo conoce. En este caso, la probabilidad de acceder a los AAI desconociendo los procedimientos precisos para hacerlo es de solo 19\%; mientras que el conocimiento que permite el acceso a estos dispositivos incrementa la posibilidad de participación al $45 \%$.

Necesidad de Ayuda Académica. Algo similar ocurre con la conciencia de necesitar ayuda en el plano académico. Una persona que es consciente de haber requerido ayuda tiene 2.25 veces más posibilidades de participar en los AAI respecto a los que declaran no haber necesitado ayuda de otros para aprender o para mejorar su rendimiento. En este caso, los primeros tienen una probabilidad de $22.6 \%$ de acceder a los AAI, mientras que los segundos un $39.7 \%$.

Costo, como pérdida de alternativas valiosas (LOVA). La variable que efectivamente actúa como inhibidor es un tipo de costo específico: el costo de perder alternativas valiosas (LOVA). En este caso, a medida que aumenta el costo disminuye la probabilidad de ser parte 
de los dispositivos de apoyo académico ofertados por la institución. Así, si el resto de las variables fueran constantes, la reducción de LOVA aumenta en 1.4 veces la probabilidad de participar en los AAI.

Así, mientras los estudiantes que se sitúan a +1 desviación estándar sobre la media de esta variable, es decir, que perciben un costo alternativo mayor, tienen una probabilidad de acceder a los AAI de $22.4 \%$, mientras que los estudiantes situados a -1 desviación estándar bajo la media perciben un menor costo en términos de pérdida de alternativas valiosa, tienen un $39.9 \%$ de probabilidades de acceder a los AAI.

Disposición a buscar ayuda en plano institucional. Finalmente, el incremento de la disposición a buscar ayuda en la institución contribuye a aumentar 1.27 veces la probabilidad de participar en estos dispositivos (AAI). Así, los estudiantes que se sitúan por debajo de la media tienen una probabilidad de participar en los AAI de un $24 \%$ mientras que para los estudiantes que se sitúan a +1 desviación por sobre la media, esta probabilidad alcanza un $37.8 \%$.

\section{Discusión y conclusiones}

El objetivo de este estudio fue evaluar la capacidad predictiva de diversas variables identificadas por la literatura asociadas a la búsqueda de ayuda general, y de otras variables que razonablemente condicionarían la búsqueda de ayuda académica institucional, sobre la probabilidad de ocurrencia de la participación de los estudiantes en los dispositivos de apoyo académico que las instituciones ofertan a sus estudiantes. Se buscó a través de un análisis de regresión identificar aquellas variables que efectivamente predicen este comportamiento y contrastar estos resultados con los antecedentes que la literatura reporta respecto a la búsqueda de ayuda en general.

Los resultados muestran que las variables que mejor predicen la búsqueda de ayuda académica de los estudiantes en el marco institucional son: a) la búsqueda previa de ayuda, b) el conocimiento de los mecanismos específicos para acceder a estos apoyos, c y d) la conciencia de dificultades académicas y de la necesidad de apoyo, e) la disposición a buscar dichos apoyos en los dispositivos ofertados por la institución y finalmente, como variable inhibitoria, f) el costo de perder alternativas valiosas. 
Así, mientras las variables antes mencionadas predicen de manera significativa esta participación, hubo otras que pese a los supuestos iniciales basados en la dinámica general de búsqueda de ayuda (Karabenick, 2014; Reeves, 2014), no son predictoras directas de la participación en los dispositivos institucionales de ayuda académica ofertados por las universidades.

Los resultados, contra lo esperado, no mostraron evidencia de un efecto predictor significativo de las variables relacionadas con el conocimiento de la existencia de apoyos académicos institucionales ofertados por la universidad; la identificación del estudiante como destinatario de esos apoyos; la valoración que los estudiantes realizan de los AAI ofertados por la institución; el compromiso académico general (Engagement);y las variables de carácter inhibitorio tales como la vergüenza y los tipos de costos asociados con el esfuerzo implicado en la tarea (Task Cost), el esfuerzo de realizar una actividad que no es de interés (Outside Effort Cost) y el costo emocional (EC). En todos estos casos el aporte predictivo no fue estadísticamente significativo $(p>.05)$.

Estos resultados tienen implicaciones teóricas. Si queremos modelizar el itinerario de la búsqueda de ayuda de un estudiante a nivel institucional, la secuencia general propuesta por Reeves (2014) o Karabenick (2014) no es suficiente. Es necesario incorporar otras variables que explicarían de manera más comprensiva este proceso de búsqueda de ayuda académica en los AAI.

Con todo, no deja de llamar la atención que el efecto de la vergüenza en contextos académicos reportados por múltiples estudios (e.g. Apto et al., 2017; Hartman, 2019; Sanchez-Rosas et al., 2016) no aparezca como predictor significativo. Una hipótesis para explicar el por qué la vergüenza no aparece como una variable predictora de la búsqueda de apoyos académicos institucionales es que, a diferencia de la búsqueda de ayuda cara a cara , donde sí se reportan efectos significativos (Reeves y Sperling, 2015), una parte importante de los apoyos académicos institucionales son colectivos. Con todo, es necesario un estudio más focalizado en el rol de esta variable en la participación en apoyos académicos institucionales.

Un segundo resultado que llama poderosamente la atención y que tiene implicancias prácticas, es que dentro de los cuatro tipos de costos (Flake et al., 2015) el único que actúa 
como predictor de la búsqueda de ayuda en AAI es el costo de perder oportunidades valiosas. La acción inhibitoria de esta variable expresa la tensión entre los apoyos académicos ofertados y otras actividades que son importantes para los estudiantes. En otras palabras, no son ni el tiempo ni el esfuerzo requeridos, variables que determinen la búsqueda de ayuda institucional. La participación en las iniciativas de apoyo académico que ofertan las instituciones estaría condicionada a que este tipo de actividad no compita con otras más valoradas por los jóvenes.

Como todo estudio, esta investigación tiene limitaciones. En el plano teórico, se carecía de estudios previos que diesen cuenta de variables predictoras de la búsqueda de ayuda en contextos institucionales. Ello limitó la posibilidad de contraste con estudios precedentes. No obstante, el carácter exploratorio de este estudio se constituye en un primer paso para comprender por qué los estudiantes podrían aprovechar o no los apoyos académicos que las instituciones diseñan e implementan para apoyar el aprendizaje en los estudios superiores.

A modo de conclusión, los resultados evidencian que las variables que tradicionalmente son consideradas como predictores importantes de la búsqueda de ayuda académica comparten un potencial explicativo con variables que actúan como condicionantes en la búsqueda de ayuda a nivel institucional. No obstante, esta aproximación preliminar al fenómeno requiere de estudios más específicos y de aproximaciones tanto cuantitativas como cualitativas que permitan dar mejor comprensión al fenómeno singular que es la búsqueda de ayuda académica. A futuro, se espera poder contar con un modelo más comprensivo que pueda dar cuenta de la interrelación estructural entre estas y otras variables y que permita testear hipótesis fuertes respecto a quiénes son los que participan en los AAI y quienes, teniendo necesidad y dadas las oportunidades, no acceden a ellos.

\section{Agradecimientos}

Esta investigación ha sido financiada por la Agencia Nacional de Investigación y Desarrollo, ANID - Chile, a través del Proyecto Fondecyt Regular Nº 1181159.

\section{Declaración de disponibilidad de datos}

Los datos que apoyan los resultados de este estudio están disponibles y pueden ser solicitados al autor correspondiente. 


\section{Referencias}

Amemiya, J., y Wang, M.-T. (2017). Transactional Relations between Motivational Beliefs and Help Seeking from Teachers and Peers across Adolescence. Journal of Youth and Adolescence, 46(8), 1743-1757. https://doi.org/10.1007/s10964-016-0623-y

Apto, M. S., Pesqueira, N. G., Vilca, B., y Sánchez-Rosas, J. (2017). Efecto y Contribución Explicativa del Apoyo e Inhibición, Interacción-Ilustración, Vergüenza, Disfrute y Amenazas a la Evitación de la Búsqueda de Ayuda Académica. Anuario de Investigaciones de la Facultad de Psicología, 3(1), 247-263. https://revistas.psi.unc.edu.ar/index.php/aifp/article/view/18670

Bornschlegl, M., Meldrum, K., y Caltabiano, N. J. (2020). Variables Related to Academic Help-Seeking Behaviour in Higher Education - Findings from a Multidisciplinary Perspective. Review of Education, 8(2), 486-522. https://doi.org/10.1002/rev3.3196

Cea D’ancona, M. (2004). Análisis multivariable. Síntesis.

Çebi, E., y Demir, A. (2020). Help-Seeking Attitudes of University Students in Turkey. International Journal for the Advancement of Counselling, 42(1), 37-47. https://doi.org/10.1007/s10447-019-09385-7

Cerda, J., Vera, C., y Rada, G. (2013). Odds ratio: aspectos teóricos y prácticos. Revista médica de Chile, 141, 1329-1335. http://dx.doi.org/10.4067/S003498872013001000014

Chowdhury, S., y Halder, S. (2019). Motivational facilitators and barriers of adaptive academic help-seeking: A systematic review. Indian Journal of Health and Wellbeing, 10(10-12), 324-333. http://www.ischolar.in/index.php/ijhw/article/view/202867

Collins, W., y Sims, B. C. (2006). Help seeking in higher education academic support services. In A. Stuart, S. Karabenick, y R. S. Newman (Eds.), Help seeking in academic settings: Goals, groups, and contexts (pp. 203-223). Reutledge.

Duchesne, S., Larose, S., y Feng, B. (2019). Achievement goals and engagement with academic work in early high school: Does seeking help from teachers matter? The Journal of Early

Adolescence, $\quad 39(2)$, 222-252. 
https://doi.org/10.1177/0272431617737626

Eccles, J. S., y Wigfield, A. (2020). From expectancy-value theory to situated expectancy-value theory: A developmental, social cognitive, and sociocultural perspective on motivation. Contemporary Educational Psychology, 101859. https://doi.org/10.1016/j.cedpsych.2020.101859

Fatoux, C., Mottet, M., y Rouissi, S. (2018). État des connaissances sur la demande d'aide: quel apport pour la formation universitaire en ligne? https://doi.org/10.18162/fp.2018.373

Finney, S. J., Barry, C. L., Horst, S. J., y Johnston, M. M. (2018). Exploring profiles of academic help seeking: A mixture modeling approach. Learning and Individual Differences, 61, 158-171. https://doi.org/10.1016/j.lindif.2017.11.011

Flake, J. K., Barron, K. E., Hulleman, C., McCoach, B. D., y Welsh, M. E. (2015). Measuring cost: The forgotten component of expectancy-value theory. Contemporary Educational Psychology, 41, 232-244. https://doi.org/10.1016/j.cedpsych.2015.03.002

Fox, J. (2016). Applied Regression Analysis and Generalized Linear Models. Sage.

Frites, C., y Molina, R. M. (2016). Tutorías y nivelación en la Universidad de Santiago: tensiones y desafíos en la implementación de iniciativas de permanencia Congresos CLABES, Medellín. https://revistas.utp.ac.pa/index.php/clabes/article/view/1045

Gonida, E. N., Karabenick, S. A., Stamovlasis, D., Metallidou, P., y the CTY Greece. (2019). Help seeking as a self-regulated learning strategy and achievement goals: The case of academically talented adolescents. High Ability Studies, 30(1-2), 147-166. https://doi.org/10.1080/13598139.2018.1535244

Hao, Q., Barnes, B., Wright, E., y Branch, R. M. (2017). The influence of achievement goals on online help seeking of computer science students. British Journal of Educational Technology, 48(6), 1273-1283. https://doi.org/10.1111/bjet.12499

Hartman, A. T. (2019). The Role of Shame in Student Persistence and Help-Seeking [Master in Education, University of Saskatchewan]. Saskatoon. https://bit.ly/3a5EPq7

Heerde, J. A., y Hemphill, S. A. (2018). Examination of associations between informal help-seeking behavior, social support, and adolescent psychosocial outcomes: A meta-analysis. Developmental Review, 47, 44-62. https://doi.org/https://doi.org/10.1016/j.dr.2017.10.001

Jaroszewicz, A. (2020). It Does Hurt to Ask: Theory and Evidence on Informal Help-Seeking 
[Doctoral Dissertation, Carnegie Mellon University]. Pittsburgh, PA. https://kilthub.cmu.edu/ndownloader/files/22668773

Karabenick, S. A. (2014). Past, present, and future of help-seeking as a self-regulated learning strategy Presentation The annual meeting of the American Educational Research Association, Philadelphia, PA.

Karabenick, S. A., y Berger, J.-L. (2013). Help seeking as a self-regulated learning strategy. In H. Bembenutty y T. J. K. Cleary, A. (Eds.), Applications of self-regulated learning across diverse disciplines: A tribute to Barry J. Zimmerman (pp. 237-261). IAP Information Age Publishing.

Karabenick, S. A., y Dembo, M. H. (2011). Understanding facilitating self-regulated help seeking. New Directions for Teaching and Learning, 2011(126), 33-43. https://doi.org/10.1002/tl.442

Karabenick, S. A., y Gonida, E. N. (2018). Academic help seeking as a self-regulated learning strategy: Current issues, future directions. In D. H. Schunk y J. A. Greene (Eds.), Handbook of self-regulation of learning and performance (pp. 421-433). Routledge/Taylor y Francis Group.

Kiefer, S. M., y Shim, S. S. (2016). Academic help seeking from peers during adolescence: The role of social goals. Journal of Applied Developmental Psychology, 42, 80-88. https://doi.org/10.1016/j.appdev.2015.12.002

Martin, A. J., Ginns, P., y Papworth, B. (2017). Motivation and engagement: Same or different? Does it matter? Learning and Individual Differences, 55, 150-162. https://doi.org/https://doi.org/10.1016/j.lindif.2017.03.013

McCabe, J. A. (2018). What learning strategies do academic support centers recommend to undergraduates? Journal of applied research in memory and cognition, 7(1), 143-153. https://doi.org/10.1016/j.jarmac.2017.10.002

Means, D. R., y Pyne, K. B. (2017). Finding my way: Perceptions of institutional support and belonging in low-income, first-generation, first-year college students. Journal of College Student Development, 58(6), 907-924. https://doi.org/10.1353/csd.2017.0071

Miranda, R., Contreras, J., Cornejo, M. F., González, F., Moris, E., y Muñoz, N. (2016). Desarrollando tus talentos: gestión colaborativa de un programa de tutorías pares Congresos CLABES, Medellín. https://revistas.utp.ac.pa/index.php/clabes/article/view/1077 
Muñoz, M., y Blanco, C. (2013). Una taxonomía de las universidades chilenas. Calidad en la educación, 181-213. http://dx.doi.org/10.31619/caledu.n38.109

Nelson-Le Gall, S. (1981). Help-seeking: An understudied problem-solving skill in children. Developmental Review, 1(3), 224-246. https://doi.org/10.1016/0273-2297(81)90019-8

Newman, R. S. (2002). How self-regulated learners cope with academic difficulty: The role of adaptive help seeking. Theory into practice, 41(2), 132-138. https://doi.org/10.1207/s15430421tip4102_10

Ordenes, C., Duran, C., y Cerda, A. b. (2018). Implementación de un sistema de tutorías académicas a nivel institucional dentro de un modelo de progresión del estudiante Congresos CLABES, Panamá. https://revistas.utp.ac.pa/index.php/clabes/article/view/1977

Osorio Vargas, M. (2019). Orientación en la Educación Superior en Chile. In Universidad de Chile (Ed.), Modelo de orientación para el aprendizaje en la educación superior (pp. 36-57). Centro de aprendizaje Campus Sur, Universidad de Chile.

Peeters, A., Robinson, V., y Rubie-Davies, C. (2020). Theories in use that explain adolescent help seeking and avoidance in mathematics. Journal of Educational Psychology, 112(3), 533. https://doi.org/10.1037/edu0000423

Pekrun, R., Elliot, A. J., y Maier, M. A. (2006). Achievement goals and discrete achievement emotions: A theoretical model and prospective test. Journal of educational Psychology, 98(3), 583. https://doi.org/10.1037/0022-0663.98.3.583

Persoskie, A., y Ferrer, R. A. (2017). A Most Odd Ratio: Interpreting and Describing Odds Ratios. American Journal of Preventive Medicine, 52(2), 224-228. https://doi.org/https://doi.org/10.1016/j.amepre.2016.07.030

Puustinen, M. (2013). La demande d'aide chez l'élève: avancées conceptuelles, méthodologiques et nouvelles données. Editions L'Harmattan.

Reeves, P. M. (2014). Academic Help Seeking Research: Exploring Future Directions. In R. Morrison (Ed.), Motivation: Psychology, Strategies and Impact on Performance (pp. 93-108). Nova Science Publishers.

Reeves, P. M., y Sperling, R. A. (2015). A comparison of technologically mediated and faceto-face help-seeking sources. $\mathrm{Br} \quad J \quad$ Educ Psychol, 85(4), 570-584. https://doi.org/10.1111/bjep.12088 
Ryan, A. M., Patrick, H., y Shim, S.-O. (2005). Differential Profiles of Students Identified by Their Teacher as Having Avoidant, Appropriate, or Dependent Help-Seeking Tendencies in the Classroom. Journal of Educational Psychology, 97(2), 275. https://doi.org/10.1037/0022-0663.97.2.275

Sanchez-Rosas, J., Takaya, P. B., y Molinari, A. V. (2016). Atención en clase: rol predictivo del comportamiento docente, valor de la tarea, autoeficacia, disfrute y vergüenza. PSIENCIA. Revista Latinoamericana de Ciencia Psicológica, 8. http://www.psiencia.org/8/3/22

Seamark, D., y Gabriel, L. (2018). Barriers to support: a qualitative exploration into the helpseeking and avoidance factors of young adults. British Journal of Guidance y Counselling, 46(1), 120-131. https://doi.org/10.1080/03069885.2016.1213372

Silva, L. C., y Barroso, I. M. (2004). Regresión logística (Vol. 27). La Muralla.

The Jamovi Proyect. (2019). Jamovi (version 1.0). In [Computer Software]. http://www.jamovi.org

Valenzuela, J., Miranda, J., González, A., y Muñoz, C. (2021). Apoyos académicos de estudiantes universitarios chilenos: Amplitud, jerarquía y estabilidad en su demanda. Formación Universitaria. 14(3), 1-12.

Venegas, L., y Gairín, J. (2020). Aproximación al estado de la acción tutorial en universidades $\begin{array}{llll}\text { chilenas. } & \text { Perfiles } & \text { 103-118. }\end{array}$ https://doi.org/10.22201/iisue.24486167e.2019.167.59002

Wigfield, A., Tonks, S. M., y Klauda, S. L. (2016). Expectancy-Value Theory In K. R. Wentzel y D. B. Miele (Eds.), Handbook of Motivation at School (pp. 67-86). Routledge. https://doi.org/10.1017/9781316823279.026

Wirtz, E., Dunford, A., Berger, E., Briody, E., Guruprasad, G., y Senkpeil, R. (2018). Resource usage and usefulness: academic help-seeking behaviours of undergraduate engineering students. Australasian Journal of Engineering Education, 23(2), 62-70. https://doi.org/10.1080/22054952.2018.1525889

Won, S., Hensley, L. C., y Wolters, C. A. (2019). Brief Research Report: Sense of Belonging and Academic Help-Seeking as Self-Regulated Learning. The Journal of Experimental Education, 1-13. https://doi.org/10.1080/00220973.2019.1703095

Zander, L., Chen, I. C., y Hannover, B. (2019). Who asks whom for help in mathematics? A 
sociometric analysis of adolescents' help-seeking within and beyond clique boundaries. Learning and Individual Differences, 72, 49-58. https://doi.org/10.1016/j.lindif.2019.03.002

Recibido: $21-10-2020$

Aceptado: $12-02-2021$ 